\title{
AI in Recruiting. Multi-agent Systems Architecture for Ethical and Legal Auditing
}

\author{
María del Carmen Fernández Martínez and Alberto Fernández \\ Artificial Intelligence Group-CETINIA, Rey Juan Carlos University, Spain \\ carmen.urjc@gmail.com, alberto.fernandez@urjc.es
}

\begin{abstract}
Artificial Intelligence (AI) domain-specific applications may have different ethical and legal implications. One of the current questions of AI is the challenges behind the analysis of job video interviews. There are pros and cons to using AI in recruitment processes, and potential consequences for candidates, companies and states. Furthermore, the deficit of regulation of these systems reinforces the need for external and neutral auditing of the types of analysis made in interviews. We, therefore, propose a Multi-agent system architecture for neutral auditing to guarantee an inclusive and accurate $\mathrm{AI}$ and to reduce the potential discrimination in the job market.
\end{abstract}

\section{Introduction}

In the early stages of the research, we focused on theoretical ontology-based information retrieval approaches to later address a current issue of the AI, such as domain-specific AI in corporate settings.

Traditionally, AI proved very valuable for resume and keywords scanning and extraction of candidate skills devoid of bias. There has been a recent trend towards videointerview analysis in Human Resources. A survey by Personnel Today found that $38 \%$ of enterprises are using $\mathrm{AI}$ in their workplace with $62 \%$ expecting to use it by $2018 .{ }^{1}$

Concerning video-interview systems, there are limitations, some are attributable to the very nature of the technology (incorrect or biased datasets), and others are related to human bias or the specific agenda of the recruiting company. It is common measuring eye time or candidate response time. However, the state-of-the-art in image analysis may allow pre-selecting for age or sexual orientation or other controversial characteristics. Such AI-based analyses could lead to ethical and legal consequences (e.g. in some countries is forbidden to ask for age in recruitment

\footnotetext{
${ }^{1}$ https://www.personneltoday.com/hr/ten-ways-hr-tech-leaderscan-make-artificial-intelligence/
}

processes). This is why fostering proper auditing of video interview systems it is particularly important.

The most relevant innovation of this proposed work will be the attempt to automatise part of this legal and ethical auditing as well as some analyses carried out by the different distributed agents. The specific contribution and progress made thus so far is mainly the proposal of a Multiagent systems architecture for auditing HR [Fig 1]. Additionally, prototyping of some parts -legal rules engine- has been created.

The future direction and goal are either the total or the partial implementation of the automatisation in the Multiagent System. Questions could arise when it comes to the complete implementation and testing of such a complex and distributed in nature system for a $\mathrm{PhD}$ thesis. Recently, we have been through the definition of the common lexicon and the full formalisation and implementation of a subset of legal rules. Both combined could help in achieving good results. We face the dilemma of formalising Labour Law and narrow it to a set of rules. For the time being, around 50 current legal rules have been considered.

Concerning limits, so far, we are in the implementation phase, more concretely defining flows among agents and prototyping. The first problem could be the use of different ontologies. As the doctoral dissertation advances, we will see if we could address in all detail the architecture or just

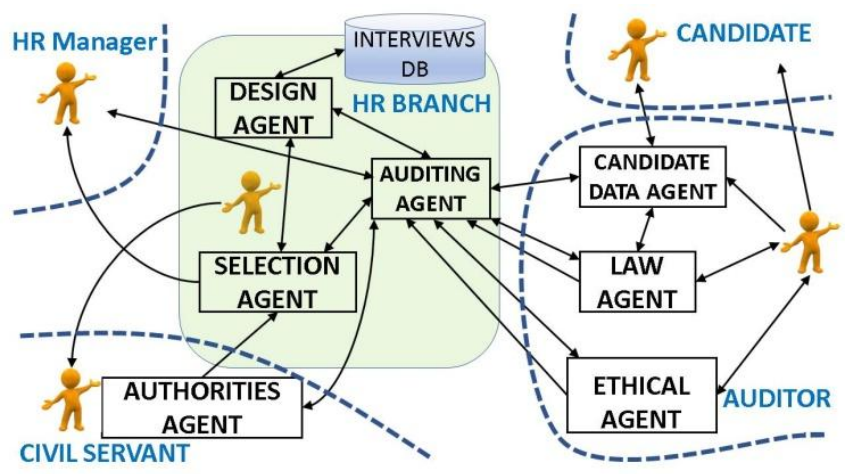

Figure 1: Multi-Agent System architecture 
the automatisation of the legal auditing if it involves hundreds of rules. Precisely, it is the absence of affordable and limited testing opportunities of real corporate applications in our academic setting which limits the research. The testing is very dependent on data availability, and the real attributed measured by video interview systems, so we are counting on prototyping on a simulated corporate scenario. Legal reasoning entails the correct formalisation of laws in a different jurisdiction and it could be necessary even the technical supervision of jurists.

The contribution of this $P h D$ is, therefore, the automatisation. The benefits of our research will be a step forward in the automatisation of tasks in HR and ethical and legal analyses of video interview techniques. To achieve this goal and solve the research problem of automatisation and more ethical and legal auditing we are going to rely on the integration of ontologies into Multi-Agent Systems for Auditing in HR. The current approach is the definition of agents tasks and the exploration of both Multi-agent systems and ontologies and shared lexicons, which foster domain specification and interoperability [Dileo et al., 2006].

Multi-agent systems pose similar challenges concerning information formats that traditional complex distributed systems and the problem is more acute in firms.

Domain-specific AI has been recently used as a decisionmaking supporting tool. A job is composed of tasks and AI in Recruiting has indeed saved tasks for managers. The trend -the aim of AI- is towards full automatisation.

\section{Motivation for the Research Paper}

There has been a recent trend towards video-interview analysis in HR departments. Traditionally, AI played no more than an assistant role in HR, e.g. resume scanning. However, lately, apps and systems like HireVue ${ }^{2}$, Montage $^{3}$, SparkHire $^{4}$ and WePow ${ }^{5}$ have been changing how recruitment is carried out. An AI-based video interview system could be programmed to check, during an interview, features such as age, lighting, tone of voice, cadence, keywords used (substantial conversation), mood, behaviour (eccentric, movement or quite calm and not talkative), eye contact and, above all, emotions. AI targets the specific traits of a customer-oriented role that employers want.

AI has produced benefits for HR so far, including recruiting time and customised questions and answers. Attention to detail (eye contact time, emotions and body language) and lack of interviewer bias (physical appearance, tattoos, etcetera) are other additional advantages. However, several problems accompany the use of these technologies; for example, candidates are unfamiliar with video interview analysis (for example, lighting, settings), which could affect global performance. It is necessary to point out the gender

\footnotetext{
${ }^{2}$ https://www.hirevue.com/

${ }^{3}$ https://www.montagetalent.com/

${ }^{4}$ https://www.sparkhire.com/

${ }^{5}$ https://www.wepow.com/es/
}

and racial bias and the imprecision of technology. Traditionally, Machine Learning algorithms were trained with biased datasets. For instance, Affectiva ${ }^{6}$ emotions dataset was fed with data from Superbowl viewers, and could presumably have culture-bias.

We studied several potential controversial characteristics, among them, facial symmetry, race, gender, sexual orientations in voice and image recordings. The problem of racial-bias in AI is not new, just like the detection of mixed race in bad lighting conditions according to Siyao et al [Siyao et al., 2014]. As an illustration of the advances in sexual orientation recognition, one study [Kosinski and Wang, 2018] needed ethical supervision due to the opaque invasive nature of the research and the use of real user data from dating applications. Researchers argue about the relationship among homosexuality, morphological features such as jawline and exposure to hormones in the womb.

Regarding Ethical and legal aspects of AI, analyses may have its benefits, it also strips away aspects of humanity, reducing a human recruit to a set of descriptors. The automation of HR processes could lead to potential ethical and legal implications that cannot be ignored. In some countries, companies are not allowed to ask a candidate's age during selection. Traditionally, United States legislation has been particularly protective of racial discrimination in the workplace (the Civil Rights Act, 1964, forbids "improperly classifying or segregating employees by race"). Moreover, yet even these regulations exist to reduce discrimination, enterprises are given more and more freedom to customise their systems. We conclude that it is risky to follow the adoption of $\mathrm{AI}$ in recruiting blindly.

\section{Acknowledgments}

Work partially supported by the Spanish Ministry of Science, Innovation and Universities, co-funded by EU FEDER Funds, through grants TIN2015-65515-C4-4-R and RTI2018-095390-B-C33.

\section{References}

[Siyao et al., 2014] Siyao Fu, Haibo He, Zeng-Guang Hou. Learning from Face: A Survey. IEEE Trans. Pattern Anal. Mach. Intel 36(12): 2483-2509, 2014.

[Kosinski and Wang, 2018] Michal Kosinksi and Yilum. Wang. Deep neural networks are more accurate than humans at detecting sexual orientation from images. Journal of Personality and Social Psychology, 114(2):246-257, 2018.

[Dileo et al., 2006] Jonathan DiLeo, Timothy Jacobs and Scott DeLoach. Integrating ontologies into multiagent systems engineering. Air Univ Maxwell AFB Al Center For Aerospace Doctrine Research and Education, 2006.

\footnotetext{
${ }^{6}$ https://www.affectiva.com/
} 